\title{
Measurement of Atmospheric Neutrino Oscillations at 6-56 GeV with IceCube DeepCore
}

M. G. Aartsen, ${ }^{2}$ M. Ackermann, ${ }^{52}$ J. Adams, ${ }^{16}$ J. A. Aguilar, ${ }^{12}$ M. Ahlers,${ }^{20}$ M. Ahrens, ${ }^{44}$ I. Al Samarai, ${ }^{25}$ D. Altmann, ${ }^{24}$ K. Andeen, ${ }^{33}$ T. Anderson, ${ }^{49}$ I. Ansseau, ${ }^{12}$ G. Anton, ${ }^{24}$ C. Argüelles, ${ }^{14}$ J. Auffenberg, ${ }^{1}$ S. Axani, ${ }^{14} \mathrm{H}$. Bagherpour, ${ }^{16}$ X. Bai, ${ }^{41}$ J. P. Barron, ${ }^{23}$ S. W. Barwick, ${ }^{27}$ V. Baum, ${ }^{32}$ R. Bay, ${ }^{8}$ J. J. Beatty, ${ }^{18,19}$ J. Becker Tjus, ${ }^{11}$ K.-H. Becker,${ }^{51}$ S. BenZvi, ${ }^{43}$ D. Berley, ${ }^{17}$ E. Bernardini, ${ }^{52}$ D. Z. Besson, ${ }^{28}$ G. Binder, ${ }^{9,8}$ D. Bindig, ${ }^{51}$ E. Blaufuss, ${ }^{17}$ S. Blot, ${ }^{52}$ C. Bohm, ${ }^{44}$ M. Börner,${ }^{21}$ F. Bos, ${ }^{11}$ D. Bose, ${ }^{46}$ S. Böser, ${ }^{32}$ O. Botner,${ }^{50}$ J. Bourbeau, ${ }^{31}$ F. Bradascio, ${ }^{52}$ J. Braun, ${ }^{31}$ L. Brayeur, ${ }^{13}$ M. Brenzke, ${ }^{1}$ H.-P. Bretz, ${ }^{52}$ S. Bron, ${ }^{25}$ J. Brostean-Kaiser, ${ }^{52}$ A. Burgman, ${ }^{50}$ T. Carver, ${ }^{25}$ J. Casey, ${ }^{31}$ M. Casier, ${ }^{13}$ E. Cheung, ${ }^{17}$ D. Chirkin, ${ }^{31}$ A. Christov, ${ }^{25}$ K. Clark, ${ }^{29}$ L. Classen, ${ }^{36}$ S. Coenders, ${ }^{35}$ G. H. Collin, ${ }^{14}$ J. M. Conrad, ${ }^{14}$ D. F. Cowen, ${ }^{49,48}$ R. Cross, ${ }^{43}$ M. Day, ${ }^{31}$ J. P. A. M. de André, ${ }^{22}$ C. De Clercq, ${ }^{13}$ J. J. DeLaunay, ${ }^{49}$ H. Dembinski, ${ }^{37}$ S. De Ridder, ${ }^{26}$ P. Desiati, ${ }^{31}$ K. D. de Vries, ${ }^{13}$ G. de Wasseige, ${ }^{13}$ M. de With, ${ }^{10}$ T. DeYoung, ${ }^{22}$ J. C. Díaz-Vélez, ${ }^{31}$ V. di Lorenzo, ${ }^{32}$ H. Dujmovic, ${ }^{46}$ J. P. Dumm,${ }^{44}$ M. Dunkman, ${ }^{49}$ B. Eberhardt, ${ }^{32}$ T. Ehrhardt, ${ }^{32}$ B. Eichmann, ${ }^{11}$ P. Eller, ${ }^{49}$ P. A. Evenson, ${ }^{37}$ S. Fahey, ${ }^{31}$ A. R. Fazely, ${ }^{7}$ J. Felde,${ }^{17}$ K. Filimonov ${ }^{8}$ C. Finley,${ }^{44}$ S. Flis ${ }^{44}$ A. Franckowiak, ${ }^{52}$ E. Friedman, ${ }^{17}$ T. Fuchs,${ }^{21}$ T. K. Gaisser,${ }^{37}$ J. Gallagher, ${ }^{30}$ L. Gerhardt, ${ }^{9}$ K. Ghorbani, ${ }^{31}$ W. Giang, ${ }^{23}$ T. Glauch, ${ }^{1}$ T. Glüsenkamp, ${ }^{24}$ A. Goldschmidt, ${ }^{9}$ J. G. Gonzalez, ${ }^{37}$ D. Grant, ${ }^{23}$ Z. Griffith, ${ }^{31}$ C. Haack, ${ }^{1}$ A. Hallgren,${ }^{50}$ F. Halzen, ${ }^{31}$ K. Hanson, ${ }^{31}$ D. Hebecker, ${ }^{10}$ D. Heereman, ${ }^{12}$ K. Helbing, ${ }^{51}$ R. Hellauer, ${ }^{17}$ S. Hickford ${ }^{51}$ J. Hignight, ${ }^{22}$ G. C. Hill, ${ }^{2}$ K. D. Hoffman, ${ }^{17}$ R. Hoffmann, ${ }^{51}$ B. Hokanson-Fasig, ${ }^{31}$ K. Hoshina, ${ }^{31, *}$ F. Huang, ${ }^{49}$ M. Huber,${ }^{35}$ K. Hultqvist, ${ }^{44}$ M. Hünnefeld, ${ }^{21}$ S. In,${ }^{46}$ A. Ishihara, ${ }^{15}$ E. Jacobi, ${ }^{52}$ G. S. Japaridze, ${ }^{5}$ M. Jeong, ${ }^{46}$ K. Jero, ${ }^{31}$ B. J. P. Jones, ${ }^{4}$ P. Kalaczynski, ${ }^{1}$ W. Kang, ${ }^{46}$ A. Kappes, ${ }^{36}$ T. Karg, ${ }^{52}$ A. Karle, ${ }^{31}$ U. Katz, ${ }^{24}$ M. Kauer, ${ }^{31}$ A. Keivani, ${ }^{49}$ J. L. Kelley, ${ }^{31}$ A. Kheirandish, ${ }^{31}$ J. Kim, ${ }^{46}$ M. Kim, ${ }^{15}$ T. Kintscher, ${ }^{52}$ J. Kiryluk, ${ }^{45}$ T. Kittler, ${ }^{24}$ S. R. Klein, ${ }^{9,8}$ G. Kohnen, ${ }^{34}$ R. Koirala ${ }^{37}$ H. Kolanoski, ${ }^{10}$ L. Köpke, ${ }^{32}$ C. Kopper, ${ }^{23}$ S. Kopper, ${ }^{47}$ J. P. Koschinsky, ${ }^{1}$ D. J. Koskinen, ${ }^{20}$ M. Kowalski, ${ }^{10,52}$ K. Krings, ${ }^{35}$ M. Kroll, ${ }^{11}$ G. Krückl, ${ }^{32}$ J. Kunnen, ${ }^{13}$ S. Kunwar, ${ }^{52}$ N. Kurahashi, ${ }^{40}$ T. Kuwabara, ${ }^{15}$ A. Kyriacou, ${ }^{2}$ M. Labare, ${ }^{26}$ J. L. Lanfranchi, ${ }^{49}$ M. J. Larson, ${ }^{20}$ F. Lauber, ${ }^{51}$ D. Lennarz, ${ }^{22}$

M. Lesiak-Bzdak, ${ }^{45}$ M. Leuermann, ${ }^{1}$ Q. R. Liu, ${ }^{31}$ L. Lu, ${ }^{15}$ J. Lünemann, ${ }^{13}$ W. Luszczak,${ }^{31}$ J. Madsen, ${ }^{42}$ G. Maggi, ${ }^{13}$ K. B. M. Mahn, ${ }^{22}$ S. Mancina,${ }^{31}$ R. Maruyama ${ }^{38}$ K. Mase, ${ }^{15}$ R. Maunu,${ }^{17}$ F. McNally,${ }^{31}$ K. Meagher, ${ }^{12}$ M. Medici, ${ }^{20}$

M. Meier, ${ }^{21}$ T. Menne, ${ }^{21}$ G. Merino, ${ }^{31}$ T. Meures, ${ }^{12}$ S. Miarecki, ${ }^{9,8}$ J. Micallef ${ }^{22}$ G. Momenté, ${ }^{32}$ T. Montaruli, ${ }^{25}$ R. W. Moore, ${ }^{23}$ M. Moulai, ${ }^{14}$ R. Nahnhauer,${ }^{52}$ P. Nakarmi, ${ }^{47}$ U. Naumann, ${ }^{51}$ G. Neer, ${ }^{22}$ H. Niederhausen, ${ }^{45}$ S. C. Nowicki, ${ }^{23}$ D. R. Nygren, ${ }^{9}$ A. Obertacke Pollmann, ${ }^{51}$ A. Olivas, ${ }^{17}$ A. O'Murchadha, ${ }^{12}$ T. Palczewski, ${ }^{9,8}$ H. Pandya, ${ }^{37}$ D. V. Pankova, ${ }^{49}$ P. Peiffer, ${ }^{32}$ J. A. Pepper, ${ }^{47}$ C. Pérez de los Heros, ${ }^{50}$ D. Pieloth, ${ }^{21}$ E. Pinat, ${ }^{12}$ M. Plum, ${ }^{33}$ P. B. Price, ${ }^{8}$ G. T. Przybylski, ${ }^{9}$ C. Raab,${ }^{12}$ L. Rädel,,${ }^{1}$ M. Rameez,${ }^{20}$ K. Rawlins, ${ }^{3}$ I. C. Rea, ${ }^{35}$ R. Reimann, ${ }^{1}$ B. Relethford,${ }^{40}$ M. Relich, ${ }^{15}$ E. Resconi, ${ }^{35}$ W. Rhode, ${ }^{21}$ M. Richman, ${ }^{40}$ S. Robertson, ${ }^{2}$ M. Rongen, ${ }^{1}$ C. Rott,${ }^{46}$ T. Ruhe, ${ }^{21}$ D. Ryckbosch, ${ }^{26}$ D. Rysewyk, ${ }^{22}$ T. Sälzer, ${ }^{1}$ S. E. Sanchez Herrera, ${ }^{23}$ A. Sandrock, ${ }^{21}$ J. Sandroos, ${ }^{32}$ S. Sarkar, ${ }^{20,39}$ S. Sarkar, ${ }^{23}$ K. Satalecka, ${ }^{52}$ P. Schlunder, ${ }^{21}$

T. Schmidt, ${ }^{17}$ A. Schneider, ${ }^{31}$ S. Schoenen, ${ }^{1}$ S. Schöneberg, ${ }^{11}$ L. Schumacher, ${ }^{1}$ D. Seckel,${ }^{37}$ S. Seunarine, ${ }^{42}$

J. Soedingrekso, ${ }^{21}$ D. Soldin, ${ }^{51}$ M. Song, ${ }^{17}$ G. M. Spiczak, ${ }^{42}$ C. Spiering, ${ }^{52}$ J. Stachurska, ${ }^{52}$ M. Stamatikos, ${ }^{18}$ T. Stanev, ${ }^{37}$ A. Stasik, ${ }^{52}$ J. Stettner, ${ }^{1}$ A. Steuer, ${ }^{32}$ T. Stezelberger, ${ }^{9}$ R. G. Stokstad, ${ }^{9}$ A. Stöß1, ${ }^{15}$ N. L. Strotjohann, ${ }^{52}$ G. W. Sullivan, ${ }^{17}$ M. Sutherland ${ }^{18}$ I. Taboada, ${ }^{6}$ J. Tatar, ${ }^{9,8}$ F. Tenholt, ${ }^{11}$ S. Ter-Antonyan, ${ }^{7}$ A. Terliuk,${ }^{52}$ G. Tešić, ${ }^{49}$ S. Tilav,${ }^{37}$ P. A. Toale, ${ }^{47}$ M. N. Tobin, ${ }^{31}$ S. Toscano, ${ }^{13}$ D. Tosi,${ }^{31}$ M. Tselengidou, ${ }^{24}$ C. F. Tung, ${ }^{6}$ A. Turcati, ${ }^{35}$ C. F. Turley, ${ }^{49}$ B. Ty, ${ }^{31}$ E. Unger, ${ }^{50}$ M. Usner, ${ }^{52}$ J. Vandenbroucke, ${ }^{31}$ W. Van Driessche,${ }^{26}$ N. van Eijndhoven, ${ }^{13}$ S. Vanheule, ${ }^{26}$ J. van Santen, ${ }^{52}$ M. Vehring, ${ }^{1}$ E. Vogel, ${ }^{1}$ M. Vraeghe, ${ }^{26}$ C. Walck, ${ }^{44}$ A. Wallace, ${ }^{2}$ M. Wallraff, ${ }^{1}$ F. D. Wandler, ${ }^{23}$ N. Wandkowsky, ${ }^{31}$ A. Waza, ${ }^{1}$ C. Weaver, ${ }^{23}$ M. J. Weiss, ${ }^{49}$ C. Wendt, ${ }^{31}$ J. Werthebach, ${ }^{21}$ S. Westerhoff, ${ }^{31}$ B. J. Whelan, ${ }^{2}$ K. Wiebe, ${ }^{32}$ C. H. Wiebusch, ${ }^{1}$ L. Wille, ${ }^{31}$ D. R. Williams, ${ }^{47}$ L. Wills,${ }^{40}$ M. Wolf, ${ }^{31}$ J. Wood, ${ }^{31}$ T. R. Wood, ${ }^{23}$ E. Woolsey, ${ }^{23}$ K. Woschnagg, ${ }^{8}$ D. L. Xu,${ }^{31}$ X. W. Xu, ${ }^{7}$ Y. Xu, ${ }^{45}$ J. P. Yanez, ${ }^{23}$ G. Yodh, ${ }^{27}$ S. Yoshida, ${ }^{15}$ T. Yuan, ${ }^{31}$ and M. Zoll ${ }^{44}$

(IceCube Collaboration) ${ }^{\dagger}$

\footnotetext{
${ }^{1}$ III. Physikalisches Institut, RWTH Aachen University, D-52056 Aachen, Germany ${ }^{2}$ Department of Physics, University of Adelaide, Adelaide 5005, Australia

${ }^{3}$ Department of Physics and Astronomy, University of Alaska Anchorage, 3211 Providence Drive, Anchorage, Alaska 99508, USA ${ }^{4}$ Department of Physics, University of Texas at Arlington,

502 Yates Street, Science Hall Room 108, Box 19059, Arlington, Texas 76019, USA ${ }^{5}$ CTSPS, Clark-Atlanta University, Atlanta, Georgia 30314, USA

${ }^{6}$ School of Physics and Center for Relativistic Astrophysics, Georgia Institute of Technology, Atlanta, Georgia 30332, USA
} 
${ }^{7}$ Department of Physics, Southern University, Baton Rouge, Louisiana 70813, USA

${ }^{8}$ Department of Physics, University of California, Berkeley, California 94720, USA

${ }^{9}$ Lawrence Berkeley National Laboratory, Berkeley, California 94720, USA

${ }^{10}$ Institut für Physik, Humboldt-Universität zu Berlin, D-12489 Berlin, Germany

${ }^{11}$ Fakultät für Physik \& Astronomie, Ruhr-Universität Bochum, D-44780 Bochum, Germany

${ }^{12}$ Université Libre de Bruxelles, Science Faculty CP230, B-1050 Brussels, Belgium

${ }^{13}$ Vrije Universiteit Brussel (VUB), Dienst ELEM, B-1050 Brussels, Belgium

${ }^{14}$ Department of Physics, Massachusetts Institute of Technology, Cambridge, Massachusetts 02139, USA

${ }^{15}$ Department of Physics and Institute for Global Prominent Research, Chiba University, Chiba 263-8522, Japan

${ }^{16}$ Department of Physics and Astronomy, University of Canterbury, Private Bag 4800, Christchurch, New Zealand

${ }^{17}$ Department of Physics, University of Maryland, College Park, Maryland 20742, USA

${ }^{18}$ Department of Physics and Center for Cosmology and Astro-Particle Physics, Ohio State University, Columbus, Ohio 43210, USA

${ }^{19}$ Department of Astronomy, Ohio State University, Columbus, Ohio 43210, USA

${ }^{20}$ Niels Bohr Institute, University of Copenhagen, DK-2100 Copenhagen, Denmark

${ }^{21}$ Department of Physics, TU Dortmund University, D-44221 Dortmund, Germany

${ }^{22}$ Department of Physics and Astronomy, Michigan State University, East Lansing, Michigan 48824, USA

${ }^{23}$ Department of Physics, University of Alberta, Edmonton, Alberta, Canada T6G 2E1

${ }^{24}$ Erlangen Centre for Astroparticle Physics, Friedrich-Alexander-Universität Erlangen-Nürnberg, D-91058 Erlangen, Germany

${ }^{25}$ Département de physique nucléaire et corpusculaire, Université de Genève, CH-1211 Genève, Switzerland

${ }^{26}$ Department of Physics and Astronomy, University of Gent, B-9000 Gent, Belgium

${ }^{27}$ Department of Physics and Astronomy, University of California, Irvine, California 92697, USA

${ }^{28}$ Department of Physics and Astronomy, University of Kansas, Lawrence, Kansas 66045, USA

${ }^{29}$ SNOLAB, 1039 Regional Road 24, Creighton Mine 9, Lively, Ontario, Canada P3Y IN2

${ }^{30}$ Department of Astronomy, University of Wisconsin, Madison, Wisconsin 53706, USA

${ }^{31}$ Department of Physics and Wisconsin IceCube Particle Astrophysics Center, University of Wisconsin, Madison, Wisconsin 53706, USA

${ }^{32}$ Institute of Physics, University of Mainz, Staudinger Weg 7, D-55099 Mainz, Germany

${ }^{33}$ Department of Physics, Marquette University, Milwaukee, Wisconsin 53201, USA

${ }^{34}$ Université de Mons, 7000 Mons, Belgium

${ }^{35}$ Physik-department, Technische Universität München, D-85748 Garching, Germany

${ }^{36}$ Institut für Kernphysik, Westfälische Wilhelms-Universität Münster, D-48149 Münster, Germany

${ }^{37}$ Bartol Research Institute and Department of Physics and Astronomy, University of Delaware, Newark, Delaware 19716, USA

${ }^{38}$ Department of Physics, Yale University, New Haven, Connecticut 06520, USA

${ }^{39}$ Department of Physics, University of Oxford, 1 Keble Road, Oxford OX1 3NP, United Kingdom

${ }^{40}$ Department of Physics, Drexel University, 3141 Chestnut Street, Philadelphia, Pennsylvania 19104, USA

${ }^{41}$ Physics Department, South Dakota School of Mines and Technology, Rapid City, South Dakota 57701, USA

${ }^{42}$ Department of Physics, University of Wisconsin, River Falls, Wisconsin 54022, USA

${ }^{43}$ Department of Physics and Astronomy, University of Rochester, Rochester, New York 14627, USA

${ }^{44}$ Oskar Klein Centre and Department of Physics, Stockholm University, SE-10691 Stockholm, Sweden

${ }^{45}$ Department of Physics and Astronomy, Stony Brook University, Stony Brook, New York 11794-3800, USA

${ }^{46}$ Department of Physics, Sungkyunkwan University, Suwon 440-746, Korea

${ }^{47}$ Department of Physics and Astronomy, University of Alabama, Tuscaloosa, Alabama 35487, USA

${ }^{48}$ Department of Astronomy and Astrophysics, Pennsylvania State University, University Park, Pennsylvania 16802, USA

${ }^{49}$ Department of Physics, Pennsylvania State University, University Park, Pennsylvania 16802, USA

${ }^{50}$ Department of Physics and Astronomy, Uppsala University, Box 516, S-75120 Uppsala, Sweden

${ }^{51}$ Department of Physics, University of Wuppertal, D-42119 Wuppertal, Germany

${ }^{52}$ DESY, D-15738 Zeuthen, Germany

(Received 25 July 2017; revised manuscript received 5 December 2017; published 13 February 2018)

We present a measurement of the atmospheric neutrino oscillation parameters using three years of data from the IceCube Neutrino Observatory. The DeepCore infill array in the center of IceCube enables the detection and reconstruction of neutrinos produced by the interaction of cosmic rays in Earth's atmosphere at energies as low as $\sim 5 \mathrm{GeV}$. That energy threshold permits measurements of muon neutrino disappearance, over a range of baselines up to the diameter of the Earth, probing the same range of $L / E_{\nu}$ as

Published by the American Physical Society under the terms of the Creative Commons Attribution 4.0 International license. Further distribution of this work must maintain attribution to the author(s) and the published article's title, journal citation, and DOI. Funded by $S C O A P^{3}$. 
long-baseline experiments but with substantially higher-energy neutrinos. This analysis uses neutrinos from the full sky with reconstructed energies from 5.6 to $56 \mathrm{GeV}$. We measure $\Delta m_{32}^{2}=2.31_{-0.13}^{+0.11} \times 10^{-3} \mathrm{eV}^{2}$ and $\sin ^{2} \theta_{23}=0.51_{-0.09}^{+0.07}$, assuming normal neutrino mass ordering. These results are consistent with, and of similar precision to, those from accelerator- and reactor-based experiments.

DOI: 10.1103/PhysRevLett.120.071801

Introduction.-It is well established that the neutrino mass eigenstates do not correspond to the neutrino flavor eigenstates, leading to flavor oscillations as neutrinos propagate through space $[1,2]$. After traveling a distance $L$, a neutrino of energy $E$ may be detected with a different flavor than it was produced with. In particular, the muon neutrino survival probability is described approximately by

$P\left(\nu_{\mu} \rightarrow \nu_{\mu}\right) \approx 1-4\left|U_{\mu 3}\right|^{2}\left(1-\left|U_{\mu 3}\right|^{2}\right) \sin ^{2}\left(\frac{\Delta m_{32}^{2} L}{4 E}\right)$,

where $U_{\mu 3}=\sin \theta_{23} \cos \theta_{13}$ is one element of the Pontecorvo-Maki-Nakagawa-Sakata $[3,4]$ matrix $U$ expressed in terms of the mixing angles $\theta_{23}$ and $\theta_{13}$, and $\Delta m_{32}^{2}=m_{3}^{2}-m_{2}^{2}$ is the splitting of the second and third neutrino mass states that drives oscillation on the length and energy scales relevant to this analysis. In addition to the parameters shown in Eq. (1), neutrino oscillations also depend on the parameters $\theta_{12}, \Delta m_{21}^{2}$, and $\delta_{\mathrm{CP}}$, but these have a negligible effect on the data presented in this paper.

Interactions of cosmic rays in the atmosphere [5-7] provide a large flux of neutrinos traveling distances ranging from $L \sim 20 \mathrm{~km}$ (vertically down-going) to $L \sim$ $1.3 \times 10^{4} \mathrm{~km}$ (vertically up-going) to a detector near the Earth's surface. For up-going neutrinos, there is complete muon neutrino disappearance at energies as high as $\sim 25 \mathrm{GeV}$. Given the density of material traversed by these neutrinos, matter effects alter Eq. (1) slightly and must be taken into account [8-11].

In this Letter, we report our measurement of $\theta_{23}$ and $\Delta m_{32}^{2}$, using the IceCube Observatory to observe oscillation-induced patterns in the atmospheric neutrino flux coming from all directions between $5.6 \mathrm{GeV}$ and $56 \mathrm{GeV}$. The results presented here complement other leading experiments [12-16] in two ways. Long-baseline experiments with baselines of a few hundred kilometers and Super-Kamiokande observe much lower-energy events [primarily charged-current quasielastic (CCQE) and resonant scattering], while our measurement relies on higherenergy deep inelastic scattering events and is thus subject to different sources of systematic uncertainty [17]. In addition, the higher-energy range of IceCube neutrinos provides complementary constraints on potential new physics in the neutrino sector [18-27].

The IceCube detector was fully commissioned in 2011, and we previously reported results [28] using data from May 2011 through April 2014. Those results were obtained using reconstruction tools that relied on unscattered Cherenkov photons and therefore were less susceptible to detector noise. The results presented here use a new reconstruction that includes scattered photons and retains an order of magnitude more events per year. Because the detector's noise rates were still stabilizing during the first year of operation, and the new reconstruction is more susceptible to noise, we chose before unblinding to use data from April 2012 through May 2015.

The IceCube DeepCore detector.-The IceCube In-Ice Array [29] is composed of 5160 downward-looking $10 \mathrm{in.}$ photomultiplier tubes (PMTs) embedded in a $1 \mathrm{~km}^{3}$ volume of the South Pole glacial ice at depths between 1.45 and $2.45 \mathrm{~km}$. The PMTs and associated electronics are enclosed in glass pressure spheres to form digital optical modules (DOMs) [30,31]. The DOMs are deployed on 86 vertical strings of 60 modules each. Of these strings, 78 are deployed in a triangular grid with horizontal spacing of about $125 \mathrm{~m}$ between strings. These DOMs are used primarily as an active veto to reject atmospheric muon events in this analysis. The remaining eight strings fill a more densely instrumented $\sim 10^{7} \mathrm{~m}^{3}$ volume of ice in the bottom center of the detector, called DeepCore, enabling detection of neutrinos with energies down to $\sim 5 \mathrm{GeV}$ [32].

Neutrino interactions in DeepCore are simulated with GENIE [33]. Hadrons produced in these interactions are simulated using GEANT4 [34], as are electromagnetic showers below $100 \mathrm{MeV}$. At higher energies, shower-toshower variation is small enough to permit the use of standardized light emission templates [35] based on GEANT4 simulations to reduce computation time. Muons' energy losses in the ice are simulated using the PROPOSAL package [36]. Cherenkov photons produced by showers and muons are tracked individually using GPU-based software to simulate scattering and absorption [37].

Reconstruction and event selection.-The event reconstruction used in this analysis models the scattering of Cherenkov photons in the ice surrounding our DOMs [38] to calculate the likelihood of the observed photoelectrons as a function of the neutrino interaction position, direction, and energy. Given the complexity of this likelihood space, the MultiNest algorithm [39] is used to find the global maximum. This reconstruction is run under two different event hypotheses: first with a $\nu_{\mu}$ charged-current (CC) interaction comprising a hadronic shower and collinear muon track emerging from the interaction vertex, and then with only a shower at the vertex (i.e., a nested 
hypothesis with zero muon track length). The latter model incorporates $\nu_{e}$ and most $\nu_{\tau}$ CC interactions as well as neutral current (NC) interactions, as we do not attempt to separate electromagnetic showers produced by a leading lepton from hadronic showers produced by the disrupted nucleus.

The $\nu_{\mu}$ CC reconstruction is used to estimate the direction and energy of the neutrino. The difference in best-fit likelihoods between the two hypotheses is used to classify our events as "track-like," if inclusion of a muon track improves the fit substantially, or "cascade-like," if the event is equally well fit without a muon. The reconstructed neutrino energy $\left(E_{\text {reco }}\right)$ distributions of events in each of these categories after final selection are shown in Fig. 1, along with the corresponding predicted distributions broken down by event type. The track-like sample is enriched in $\nu_{\mu}$ CC events (68\% of sample), especially at higher energies where muons are more likely detected, while the cascade-like sample is evenly divided between $\nu_{\mu} \mathrm{CC}$ and interactions without a muon in the final state. The angular and energy resolutions provided by the reconstruction are energy dependent, with median resolutions of $10^{\circ}\left(16^{\circ}\right)$ in zenith angle and 24\% (29\%) in neutrino energy for tracklike (cascade-like) events at $E_{\nu}=20 \mathrm{GeV}$.

The event selection in this analysis uses the DOMs surrounding the DeepCore region to veto atmospheric muons. The first criteria remove accidental triggers caused by dark noise by demanding a minimum amount of light detected in the DeepCore volume, with timing and spatial scale consistent with a particle emitting Cherenkov radiation. Events in which photons are observed outside the

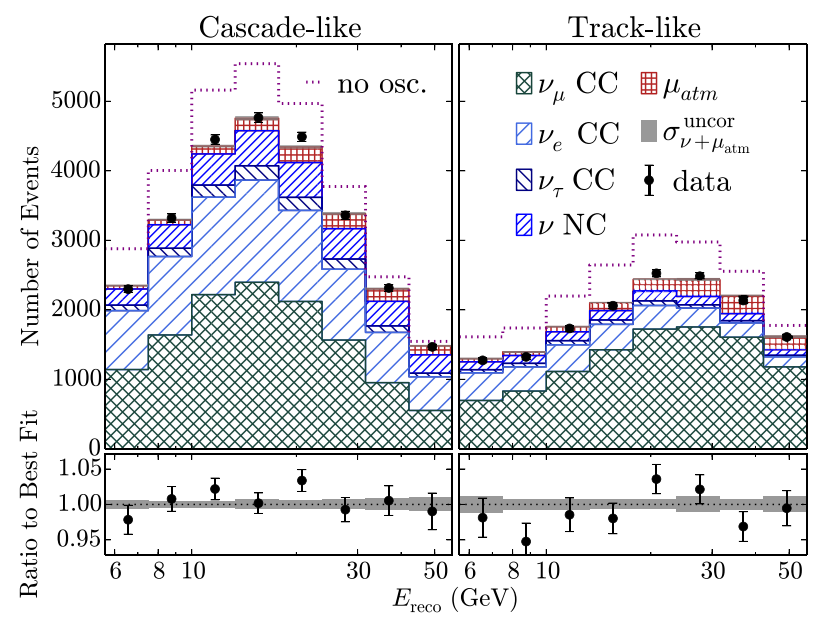

FIG. 1. Reconstructed energy distributions observed in data (points) and predicted by interaction type at our best-fit point for oscillations (stacked). In addition to each separate component, the uncorrelated statistical uncertainty associated with the expectation $\left(\sigma_{\nu+\mu_{\text {atm }}}^{\mathrm{uncor}}\right)$ is shown in a shaded band. The track-like sample is peaked at higher energy due to the rising probability of tagging $\nu_{\mu}$ $\mathrm{CC}$ events. The bottom plots show the ratio of the data to the fitted prediction.
DeepCore volume before the light detected inside DeepCore, in a time window consistent with atmospheric muons penetrating to the fiducial volume, are then rejected. These are followed by a boosted decision tree (BDT) [40] which further reduces the background of atmospheric muons. The BDT uses the timing and spatial scale of the detected photoelectrons to select events with substantial charge deposition at the beginning of the event, indicative of a neutrino interaction vertex. It also considers how close the event is to the border of the DeepCore volume and the results of several fast directional reconstructions [41] in determining whether the event may be an atmospheric muon. Finally, we demand that the interaction vertex reconstructed by the likelihood fit described above be contained within DeepCore and that the end of the reconstructed muon be within the first row of DOMs outside DeepCore, which further reduces atmospheric muon contamination and improves reconstruction accuracy.

As these selection criteria reduce the atmospheric muon rate by a factor of approximately $10^{8}$, it is challenging to simulate enough atmospheric muons to obtain a reliable prediction for the distribution of the remaining muons, especially in the presence of systematic uncertainties. We instead use a data-driven estimate of the shape of the muon background distributions, with the normalization free to float. This approach is based on tagging events that would have been accepted except for a small number of photons detected in the veto region, similar to the procedure in Ref. [28]. The uncertainty in the background shape is estimated using two different criteria for tagging these events, and was compared to the currently available muon Monte Carlo simulations. This uncertainty is added in quadrature to the statistical uncertainties in the tagged background event sample and the neutrino Monte Carlo simulations, to provide the total uncorrelated statistical uncertainty $\left(\sigma_{\nu+\mu_{\text {atm }}}^{\text {uncor }}\right)$ in the expected distribution shown in Fig. 1.

Analysis.-The final fit of the data is done using an $8 \times 8 \times 2$ binned histogram, with eight bins in $\log _{10} E_{\text {reco }}$, eight bins in the cosine of the reconstructed neutrino zenith direction $\left(\cos \theta_{z \text {,reco }}\right)$, one track-like bin, and one cascadelike bin. The bins are equally spaced with $\cos \theta_{z \text {,reco }} \in$ $[-1,1]$ and $\log _{10} E_{\text {reco }} \in[0.75,1.75]$. The fit assumes three-flavor oscillations with $\Delta m_{21}^{2}=7.53 \times 10^{-5} \mathrm{eV}^{2}$, $\sin ^{2} \theta_{12}=0.304, \sin ^{2} \theta_{13}=2.17 \times 10^{-2}$, and $\delta_{\mathrm{CP}}=0^{\circ}$.

We use MINUIT2 [42] to minimize a function

$\chi^{2}=\sum_{i \in\{\text { bins }\}} \frac{\left(n_{i}^{\nu+\mu_{\text {atm }}}-n_{i}^{\text {data }}\right)^{2}}{\left(\sigma_{i}^{\text {data }}\right)^{2}+\left(\sigma_{\nu+\mu_{\text {atm }}, i}^{\text {uncor }}\right)^{2}}+\sum_{j \in\{\text { syst }\}} \frac{\left(s_{j}-\hat{s}_{j}\right)^{2}}{\hat{\sigma}_{s_{j}}^{2}}$,

where $n_{i}^{\nu+\mu_{\text {atm }}}$ is the number of events expected in the $i$ th bin, which is the sum of neutrino events weighted to the desired oscillation parameters using PROB3++ [43] and the atmospheric muon background. The number of events observed in the $i$ th bin is $n_{i}^{\text {data }}$, with Poisson uncertainty 
$\sigma_{i}^{\mathrm{data}}=\sqrt{n_{i}^{\mathrm{data}}}$, and $\sigma_{\nu+\mu_{\mathrm{atm}}, i}^{\mathrm{unco}}$ is the uncertainty in the prediction of the number of events of the $i$ th bin. $\sigma_{\nu+\mu_{\text {atm }}}^{\text {uncor }}$ includes both effects of finite MC statistics and uncertainties in our data-driven muon background estimate. The second term of Eq. (2) is a penalty term for our nuisance parameters, where $s_{j}$ is the value of the $j$ th systematic, $\hat{s}_{j}$ is the central value, and $\hat{\sigma}_{s_{j}}^{2}$ is the Gaussian width of the $j$ th systematic prior.

The analysis includes 11 nuisance parameters describing our systematic uncertainties, summarized in Table I. Seven of these are related to systematic uncertainties in the atmospheric neutrino flux and interaction cross sections. Since only the event rate is observed directly, some uncertainties in flux and cross section have similar effects on the data. In these cases, the degenerate effects are combined into a single parameter. Because analytical models of these effects are available, these parameters can be varied continuously by reweighting simulated events.

The first nuisance parameter is the overall normalization of the event rate. It is affected by uncertainties in the atmospheric neutrino flux and the neutrino interaction cross section, and by the possibility of accidentally vetoing neutrino events due to unrelated atmospheric muons detected in the veto volume. This last effect is expected to reduce the neutrino rate by several percent, but it is not included in the present simulations. Because of this and the fact that it encompasses several effects, no prior is used for this parameter.

TABLE I. Table of nuisance parameters along with their associated priors, if applicable. The two rightmost columns show the results from our best fit for normal mass ordering and inverted mass ordering, respectively.

\begin{tabular}{|c|c|c|c|}
\hline \multirow[b]{2}{*}{ Parameters } & \multirow[b]{2}{*}{ Priors } & \multicolumn{2}{|c|}{ Best fit } \\
\hline & & NO & $\mathrm{IO}$ \\
\hline \multicolumn{4}{|c|}{ Flux and cross-section parameters } \\
\hline Neutrino event rate [\% of nominal] & No prior & 85 & 85 \\
\hline$\Delta \gamma($ spectral index $)$ & $0.00 \pm 0.10$ & -0.02 & -0.02 \\
\hline$M_{A}($ resonance $)[\mathrm{GeV}]$ & $1.12 \pm 0.22$ & 0.92 & 0.93 \\
\hline$\nu_{e}+\bar{\nu}_{e}$ relative normalization [\%] & $100 \pm 20$ & 125 & 125 \\
\hline $\mathrm{NC}$ relative normalization $[\%]$ & $100 \pm 20$ & 106 & 106 \\
\hline $\begin{array}{l}\text { Hadronic flux, energy } \\
\text { dependent }[\sigma]\end{array}$ & $0.00 \pm 1.00$ & -0.56 & -0.59 \\
\hline $\begin{array}{l}\text { Hadronic flux, zenith } \\
\text { dependent }[\sigma]\end{array}$ & $0.00 \pm 1.00$ & -0.55 & -0.57 \\
\hline \multicolumn{4}{|c|}{ Detector parameters } \\
\hline Overall optical efficiency [\%] & $100 \pm 10$ & 102 & 102 \\
\hline $\begin{array}{l}\text { Relative optical efficiency, } \\
\text { lateral }[\sigma]\end{array}$ & $0.0 \pm 1.0$ & 0.2 & 0.2 \\
\hline $\begin{array}{l}\text { Relative optical efficiency, } \\
\text { head-on [a.u.] }\end{array}$ & No prior & -0.72 & -0.66 \\
\hline \multicolumn{4}{|c|}{ Background } \\
\hline $\begin{array}{l}\text { Atm. } \mu \text { contamination } \\
{[\% \text { of sample }]}\end{array}$ & No prior & 5.5 & 5.6 \\
\hline
\end{tabular}

A second parameter allows an energy-dependent shift in the event rate. This can arise from uncertainties in either the spectral index of the atmospheric flux (nominally $\gamma=-2.66$ at the relevant energies in our neutrino flux model [7]) or the deep inelastic scattering (DIS) cross section. A prior of $\hat{\sigma}_{s}=0.10$ is placed on the spectral index to describe the range of these uncertainties.

Several uncertainties on the DIS cross section were implemented in the fit, but found either to have negligible impact or to be highly degenerate with the normalization and spectral index parameters over the energy range of this analysis. These include values of parameters of the BodekYang model [44] used in GENIE, uncertainties in the differential DIS cross section, and hadronization uncertainties for high- $W$ DIS events [45]. As these effects are captured by the first two nuisance parameters, the additional parameters were not used.

One neutrino cross-section uncertainty was not well described by these parameters: the uncertainty of the axial mass form factor for resonant events. The default value of $1.12 \mathrm{GeV}$ and prior of $0.22 \mathrm{GeV}$ were taken from GENIE [33]. Uncertainties in CCQE interactions were also investigated but had no impact on the analysis due to the small percentage of CCQE events at these energies.

The normalizations of $\nu_{e}+\bar{\nu}_{e}$ events and NC events, defined relative to $\nu_{\mu}+\bar{\nu}_{\mu} \mathrm{CC}$ events, are both assigned an uncertainty of $20 \%$. Uncertainties in hadron production (especially pions and kaons) in air showers affect the predicted flux - in particular, the ratio of neutrinos to antineutrinos. We model these hadronic flux effects with two parameters, one dependent on neutrino energy and the other on the zenith angle, chosen to reproduce the uncertainties estimated in Ref. [46]. Their total uncertainty varies from $3 \%$ to $10 \%$ depending on the energy and zenith angle, so the fit result is given in units of $\sigma$ as calculated by Barr et al. Uncertainties in the relative cross section of neutrinos versus antineutrinos are degenerate with the flux uncertainty in this energy range.

Systematics related to the response of the detector itself, including photon propagation through the ice and the anisotropic sensitivity of the DOMs, have the largest impact on this analysis. Their effects are estimated by Monte Carlo simulation at discrete values, with the contents of each bin in the (energy, direction, track or cascade) analysis histogram determined by linear interpolation between the discrete simulated models, following the approach of Refs. [27,28].

Uncertainties in the efficiency of photon detection are driven by the formation of bubbles in the refrozen ice columns in the holes where the IceCube strings were deployed. A prior with a width of $10 \%$ was applied to the overall photon collection efficiency [29], parametrized using seven MC data sets ranging from $88 \%$ to $112 \%$ of the nominal optical efficiency. In addition to modifying the absolute efficiency, these bubbles can scatter Cherenkov 
photons near the DOMs, modulating the relative optical efficiency as a function of the incident photon angle. The effect of the refrozen ice column is modeled by two effective parameters controlling the shape of the DOM angular acceptance curve.

The first parameter controls the lateral angular acceptance (i.e., relative sensitivity to photons traveling roughly $20^{\circ}$ above versus below the horizontal) and is fairly well constrained by LED calibration data. Five MC data sets were generated covering the $-1 \sigma$ to $+1 \sigma$ uncertainty from the LED calibration, and were parametrized in the same way as the overall optical efficiency described above. A Gaussian prior based on the LED data is used.

The second parameter controls sensitivity to photons traveling vertically upward and striking the DOMs head on, which is not well constrained by string-to-string LED calibration. That effect is modeled using a dimensionless parameter ranging from -5 (corresponding to a bubble column completely obscuring the DOM face for vertically incident photons) to 2.5 (no obscuration). Zero corresponds to constant sensitivity for angles of incidence from $0^{\circ}$ to $30^{\circ}$ from vertical. Six MC sets covering the range from -5 to 2 were used to parametrize this effect. No prior is applied to this parameter due to lack of information from calibration data.

The last nuisance parameter controls the level of atmospheric muon contamination in the final sample. As described above, the shape of this background in the analysis histogram, including binwise uncertainties, is derived from data. Since the absolute efficiency for tagging background events with this method is unknown, the normalization of the muon contribution is left free in the fit.

An illustration of how these nuisance parameters are constrained in the fit is provided as Supplemental Material [47]to this Letter. In addition to the systematic uncertainties discussed above, we have considered the impact of seed dependence in our event reconstruction, different optical models for both the undisturbed ice and the refrozen ice columns, and an improved detector calibration currently being prepared. In all these cases the impact on the final result was found to be minor, and they were thus omitted from the fit and the error estimate.

Results and conclusion.-The analysis procedure described above gives a best fit of $\Delta m_{32}^{2}=2.31_{-0.13}^{+0.11} \times$ $10^{-3} \mathrm{eV}^{2}$ and $\sin ^{2} \theta_{23}=0.51_{-0.09}^{+0.07}$, assuming normal neutrino mass ordering (NO). For the inverted mass ordering (IO), the best fit shifts to $\Delta m_{32}^{2}=-2.32 \times 10^{-3} \mathrm{eV}^{2}$ and $\sin ^{2} \theta_{23}=0.51$. The pulls on the nuisance parameters are shown in Table I. Though IceCube's current sensitivity to the mass ordering is low, dedicated analyses are underway to measure this.

The data agree well with the best-fit MC data set, with $\chi^{2}=117.4$ for both neutrino mass orderings. This corresponds to a $p$ value of 0.52 given the 119 effective degrees of freedom estimated via toy MCs, following the procedure described in Ref. [27].

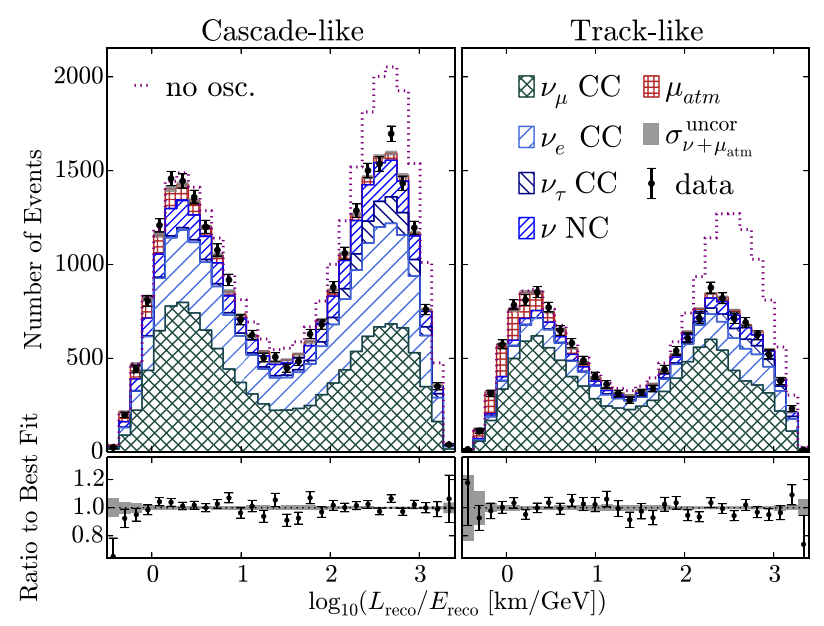

FIG. 2. Data projected onto $L / E$ for illustration. The black dots indicate the data along with their corresponding statistical errors. The dotted line shows the expectation in the absence of neutrino oscillations. The stacked hatched histograms are the predicted counts given the best-fit values of all parameters in the fit for each component. The bottom plots show the ratio of the data to the fitted prediction. The bars indicate statistical uncertainties, and the shaded region corresponds to the $\sigma_{\nu+\mu_{\text {atm }}}^{\text {uncor }}$ uncertainty in the expectation, as defined in Eq. (2), which is dominated by the uncertainty in $\mu_{\mathrm{atm}}$.

To better visualize the fit, Fig. 2 shows the results of the fit projected onto a single $L / E$ axis, for both the track-like and cascade-like events. The two peaks in each distribution correspond to down-going and up-going neutrino trajectories. Up-going $\nu_{\mu}+\bar{\nu}_{\mu}$ are strongly suppressed in the track-like channel due to oscillations. Some suppression of up-going cascade-like data is also visible, due to the disappearance of lower-energy $\nu_{\mu}$ which are not tagged as track-like by our reconstruction.

Figure 3 shows the region of $\sin ^{2} \theta_{23}$ and $\Delta m_{32}^{2}$ allowed by our analysis at $90 \%$ C.L., along with our best fit and several other leading measurements of these parameters $[12-14,16]$. The contours are calculated using the approach of Feldman and Cousins [48] to ensure proper coverage.

Our results are consistent with those from other experiments [12-16], but using significantly higher-energy neutrinos and subject to a different set of systematic uncertainties. Our data prefer maximal mixing, similar to the result from $\mathrm{T} 2 \mathrm{~K}$ [13]. The best-fit values from the $\mathrm{NO} \nu \mathrm{A}$ experiment [14] are disfavored by $\Delta \chi^{2}=8.9$ (first octant) or $\Delta \chi^{2}=8.8$ (second octant), corresponding to a significance of $2.6 \sigma$ using the method of Feldman and Cousins, although there is considerable overlap in the 90\% confidence regions of the two measurements. Further improvements to our analysis are underway, including the incorporation of additional years of data, extensions of our event selections, and improved calibration of the detector response. 


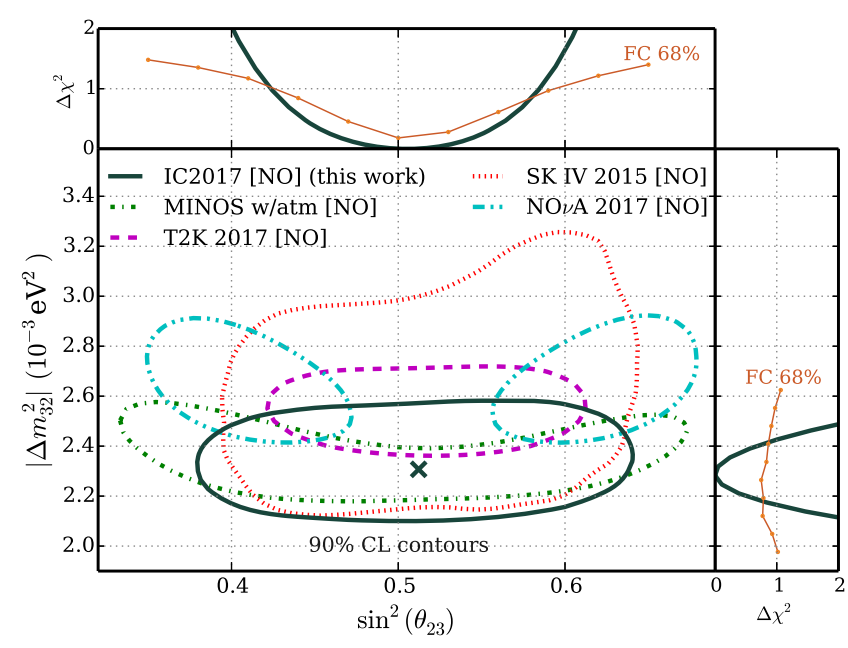

FIG. 3. The $90 \%$ allowed region from this work (solid line) compared to other experiments [12-14,16] (dashed lines). The cross marks our best-fit point. The outer plots show the results of the $1 \mathrm{D}$ projections after profiling over the other variables along with the $68 \%$ C.L. $\Delta \chi_{c}^{2}$ threshold estimated using the FeldmanCousins method [48].

We acknowledge the support from the following agencies: the U.S. National Science FoundationOffice of Polar Programs, U.S. National Science Foundation-Physics Division, the University of Wisconsin Alumni Research Foundation, Michigan State University, the Grid Laboratory Of Wisconsin (GLOW) grid infrastructure at the University of Wisconsin-Madison, and the Open Science Grid (OSG) grid infrastructure; the U.S. Department of Energy and National Energy Research Scientific Computing Center, and the Louisiana Optical Network Initiative (LONI) grid computing resources; the Natural Sciences and Engineering Research Council of Canada, WestGrid and Compute/Calcul Canada; the Swedish Research Council, Swedish Polar Research Secretariat, Swedish National Infrastructure for Computing (SNIC), and Knut and Alice Wallenberg Foundation, Sweden; the German Ministry for Education and Research (BMBF), Deutsche Forschungsgemeinschaft (DFG), Helmholtz Alliance for Astroparticle Physics (HAP), and Initiative and Networking Fund of the Helmholtz Association, Germany; the Fund for Scientific Research (FNRSFWO), FWO Odysseus programme, Flanders Institute to encourage scientific and technological research in industry (IWT), and Belgian Federal Science Policy Office (Belspo); the Marsden Fund, New Zealand; the Australian Research Council; the Japan Society for Promotion of Science (JSPS); the Swiss National Science Foundation (SNSF), Switzerland; the National Research Foundation of Korea (NRF); and Villum Fonden, Danish National Research Foundation (DNRF), Denmark.
*Also at Earthquake Research Institute, University of Tokyo, Bunkyo, Tokyo 113-0032, Japan. †analysis@icecube.wisc.edu

[1] Y. Fukuda et al. (Super-Kamiokande Collaboration), Phys. Rev. Lett. 81, 1562 (1998).

[2] Q. R. Ahmad et al. (SNO Collaboration), Phys. Rev. Lett. 87, 071301 (2001).

[3] B. Pontecorvo, Zh. Eksp. Teor. Fiz. 33, 549 (1957) [Sov. Phys. JETP 6, 429 (1957)].

[4] Z. Maki, M. Nakagawa, and S. Sakata, Prog. Theor. Phys. 28, 870 (1962).

[5] L. V. Volkova, Yad. Fiz. 31, 1510 (1980) [Sov. J. Nucl. Phys. 31, 784 (1980)].

[6] G. D. Barr, T. K. Gaisser, P. Lipari, S. Robbins, and T. Stanev, Phys. Rev. D 70, 023006 (2004).

[7] M. Honda, M. S. Athar, T. Kajita, K. Kasahara, and S. Midorikawa, Phys. Rev. D 92, 023004 (2015).

[8] L. Wolfenstein, Phys. Rev. D 17, 2369 (1978).

[9] S. T. Petcov, Phys. Lett. B 434, 321 (1998).

[10] E. K. Akhmedov, M. Maltoni, and A. Yu. Smirnov, J. High Energy Phys. 05 (2007) 077.

[11] E. K. Akhmedov, M. Maltoni, and A. Yu. Smirnov, J. High Energy Phys. 06 (2008) 072.

[12] P. Adamson et al. (MINOS Collaboration), Phys. Rev. Lett. 110, 251801 (2013).

[13] K. Abe et al. (T2K Collaboration), Phys. Rev. Lett. 118, 151801 (2017).

[14] P. Adamson et al. (NO $\nu$ A Collaboration), Phys. Rev. Lett. 118, 151802 (2017).

[15] F. P. An et al. (Daya Bay Collaboration), Phys. Rev. D 95, 072006 (2017).

[16] R. Wendell (Super-Kamiokande Collaboration), AIP Conf. Proc. 1666, 100001 (2015).

[17] J. A. Formaggio and G. P. Zeller, Rev. Mod. Phys. 84, 1307 (2012).

[18] A. Friedland, C. Lunardini, and M. Maltoni, Phys. Rev. D 70, 111301 (2004).

[19] A. Friedland and C. Lunardini, Phys. Rev. D 72, 053009 (2005).

[20] T. Ohlsson, H. Zhang, and S. Zhou, Phys. Rev. D 88, 013001 (2013).

[21] A. Esmaili and A. Yu. Smirnov, J. High Energy Phys. 06 (2013) 026.

[22] M. C. Gonzalez-Garcia and M. Maltoni, J. High Energy Phys. 09 (2013) 152.

[23] I. Mocioiu and W. Wright, Nucl. Phys. B893, 376 (2015).

[24] S. Choubey and T. Ohlsson, Phys. Lett. B 739, 357 (2014).

[25] P. Coloma and T. Schwetz, Phys. Rev. D 94, 055005 (2016); 95, 079903(E) (2017).

[26] J. Liao, D. Marfatia, and K. Whisnant, Phys. Rev. D 93, 093016 (2016).

[27] M. G. Aartsen et al. (IceCube Collaboration), Phys. Rev. D 95, 112002 (2017).

[28] M. G. Aartsen et al. (IceCube Collaboration), Phys. Rev. D 91, 072004 (2015).

[29] M. G. Aartsen et al. (IceCube Collaboration), J. Instrum. 12, P03012 (2017).

[30] R. Abbasi et al. (IceCube Collaboration), Nucl. Instrum. Methods Phys. Res., Sect. A 601, 294 (2009). 
[31] R. Abbasi et al. (IceCube Collaboration), Nucl. Instrum. Methods Phys. Res., Sect. A 618, 139 (2010).

[32] R. Abbasi et al. (IceCube Collaboration), Astropart. Phys. 35, 615 (2012).

[33] C. Andreopoulos et al., Nucl. Instrum. Methods Phys. Res., Sect. A 614, 87 (2010).

[34] S. Agostinelli et al. (GEANT4 Collaboration), Nucl. Instrum. Methods Phys. Res., Sect. A 506, 250 (2003).

[35] L. Radel and C. Wiebusch, Astropart. Phys. 38, 53 (2012).

[36] J. H. Koehne, K. Frantzen, M. Schmitz, T. Fuchs, W. Rhode, D. Chirkin, and J. B. Tjus, Comput. Phys. Commun. 184, 2070 (2013).

[37] C. Kopper et al., https://github.com/claudiok/clsim.

[38] M. G. Aartsen et al. (IceCube Collaboration), Nucl. Instrum. Methods Phys. Res., Sect. A 711, 73 (2013).

[39] F. Feroz, M. P. Hobson, and M. Bridges, Mon. Not. R. Astron. Soc. 398, 1601 (2009).
[40] A. Hocker et al., Proc. Sci., ACAT2007 (2007) 040.

[41] J. Ahrens et al. (AMANDA Collaboration), Nucl. Instrum. Methods Phys. Res., Sect. A 524, 169 (2004).

[42] F. James and M. Roos, Comput. Phys. Commun. 10, 343 (1975).

[43] R. Wendell et al., http://www.phy.duke.edu/ raw22/public/ Prob3++.

[44] A. Bodek and U. K. Yang, J. Phys. G 29, 1899 (2003).

[45] T. Katori, P. Lasorak, S. Mandalia, and R. Terri, J. Phys. Soc. Jpn. Conf. Proc. 12, 010033 (2016).

[46] G. D. Barr, S. Robbins, T. K. Gaisser, and T. Stanev, Phys. Rev. D 74, 094009 (2006).

[47] See Supplemental Material at http://link.aps.org/ supplemental/10.1103/PhysRevLett.120.071801 for additional details regarding the treatment of systematics in this analysis.

[48] G. J. Feldman and R. D. Cousins, Phys. Rev. D 57, 3873 (1998). 